\title{
Experimental Characterization of the Body-Coupled Communications Channel
}

\author{
Tim C. W. Schenk, Nafiseh Seyed Mazloum, Luc Tan, Peter Rutten \\ Philips Research, Distributed Sensor Systems department, High Tech Campus 37, 5656 AE Eindhoven, The Netherlands, \\ Tel. +31 4027 49764, Fax. +31 4027 46276, tim.schenk@philips.com
}

\begin{abstract}
Body-coupled communications (BCC), in which the human body is used as a communications channel, has been shown to be a promising solution for wireless body-area networks (WBANs). For successful deployment of these BCC-based WBANs, it is necessary to develop a clear understanding of the channel behavior. Therefore, this paper presents the key characteristics of the capacitively-coupled on-body channel used for BCC. This is based on an experimental study, which was carried out with a specifically designed measurement system. The goal of the study was to reveal the influence of electrode design, electrode position and body motion on the propagation loss and to characterize the experienced interference. It is concluded that the maximum propagation loss for the whole body channel is below $80 \mathrm{~dB}$. Moreover, the frequency dispersion and the influence of body movement on channel attenuation are shown to be much smaller than for radio frequency (RF) WBAN channels. From the results we conclude that $\mathrm{BCC}$ can result in a simpler, more robust, and lower-power WBAN than what is achievable with traditional RF solutions.
\end{abstract}

\section{INTRODUCTION}

With the growing number of mobile electronic devices surrounding a person's body, there is an increasing need to wirelessly connect these devices into a wireless bodyarea network (WBAN). For unobtrusive medical monitoring, WBANs are a key enabling technology. This field includes amongst others vital sign monitoring in hospitals, remote monitoring of chronically ill patients and assisted living of elderly at home [1]. For all these applications, several sensors will be placed on the human body, which need to communicate with each other. Key design parameters for such WBANs are reliability, power efficiency and security.

Traditionally radio frequency (RF) communications are proposed as basis for WBANs, but recently approaches have been presented in literature that use the human body itself as propagation medium [2], [3]. One advantage of this technique, which we will refer to as body-coupled communications (BCC), is that full coverage is provided, while at the same time the communications range is limited to the close proximity of the human body. Consequently, only communication between nodes close to or on the same human body is enabled, which largely prevents interference between BCC-based WBANs. This results in a frequency reuse factor of close to 1, i.e. every WBAN can use the same frequency band. This is in contrast to RF WBANs, where limited body coverage due to body shadowing, see e.g [4], [5], and interference between different WBANs, see e.g. [6], [7], are serious challenges. Recently, it has been shown that BCC devices can be implemented to use extremely low power [8]. This is enabled by the fact that BCC devices do not require power consuming high-frequency frontends, since the frequency band of interest for BCC operation lies below $100 \mathrm{MHz}$.

The different approaches proposed in literature for BCC can basically be divided in two groups: a galvanic transmission line approach and a differential capacitive coupling approach. For the first approach direct skin contact is required, which is not the case for the capacitive approach. For that reason the capacitive approach is preferred and considered here.

To design a suitable BCC solution for WBANs, a good understanding of the on-body channel is essential. Recently, some papers have described BCC channel characterization efforts. The galvanic coupled on-body channel is discussed in [9]. In other contributions, see e.g. [10]-[12], some aspects of the channel for capacitive coupling have been presented. In these works, however, the transmitter (TX) and/or receiver (RX) of the setup were connected to the earth ground via a mains connection, making the characterized channel different from the one experienced during WBAN operation. Moreover, the number of configurations considered in these papers is limited.

This paper extends previous literature by characterizing the capacitive on-body channel more extensively. The measurement results for the $\mathrm{BCC}$ channel presented are obtained using a specifically designed measurement system, in which both the TX and RX have no earth ground connection. This better resembles the actual situation of a WBAN than in previous approaches found in the literature. The influence of different system aspects has been characterized, including positions of couplers on the body, dimensions of the coupler and the influence of body movement and interference.

The outline of this paper is as follows. First we describe the developed measurement setup in Section II. Then the results from the propagation loss, body movement and interference characterization are presented in Sections III, IV and V, respectively. These sections also discuss the impact of the results on WBAN system design and compare the BCC results with previous results for RF-based WBANs. Finally, the paper ends with conclusions in Section VI.

\section{Measurement Setup}

We developed a measurement system to characterize the WBAN BCC channel. Both the TX and RX are isolated from each other and from any device connected or heavily coupled 


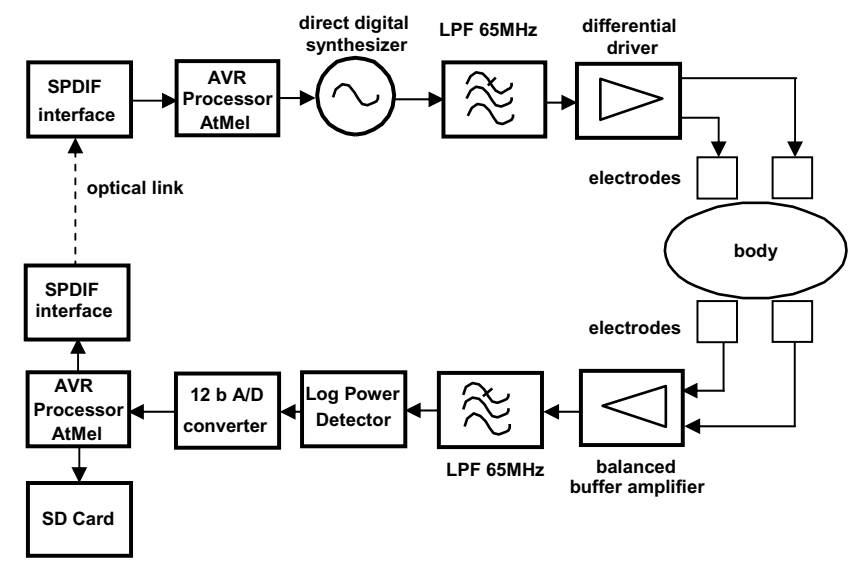

Fig. 1. Functional block diagram of the measurement system.

to the earth ground. The galvanic isolation from earth ground is achieved by making both TX and RX battery-fed and by connecting them only by an optical cable. The functional block diagram of the system is depicted in Fig. 1, where the top and bottom part represent the TX and RX respectively.

The TX consists of a direct-digital synthesizer (DDS) used as a tunable frequency source to sweep the frequency band of interest, i.e. $100 \mathrm{kHz}$ to $60 \mathrm{MHz}$. Higher frequencies were not considered since it was previously shown that at these frequencies the human body acts as an antenna and the communications is no longer limited to the human body. Control words received over the S/PDIF-based optical link, which is used for TX-RX synchronization, are interpreted by the microprocessor and are used to determine the corresponding setting for the DDS. The output of the DDS is filtered and amplified before being transmitted over the body via the coupler, consisting of two differentially driven electrodes. The TX output power is approximately $10 \mathrm{dBm}$.

The high input impedance RX basically consists of a power detector to measure power at the different transmitted frequencies. The signal received by the coupler is amplified and passed through a filter which is tuned to the measurement range to limit the interference and noise contributions. Then the power of the filtered signal is estimated by a logarithmic power detector, the output of which is sampled by a 12bit analogue-to-digital (A/D) convertor. The microprocessor temporarily stores these measurement results. Subsequently, it requests the TX to transmit the signal for the next frequency. After a set of measurements is performed, the data stored in the microprocessor memory is written onto a SD-memory card to be analyzed off-line. Using a back-to-back calibration measurement, the power measurement results can be translated into propagation loss between the TX and RX couplers.

We note that the input impedance of the $\mathrm{RX}$ is of prime importance for the characterization of the BCC channel. BCC devices are $\mathrm{AC}$ coupled to the human body and this capacitive effect, that is in series with the input of the receiver system, together with the input impedance of the device, provides a high-pass filtering of the incoming signal. For this reason the

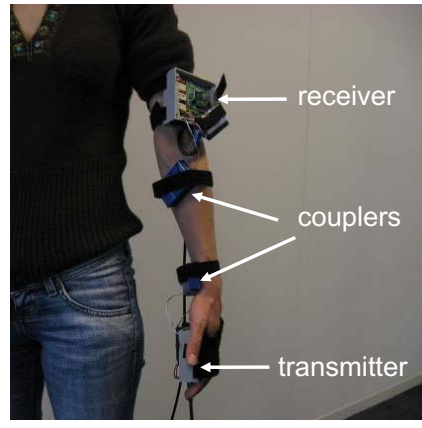

(a) system attached to the arm at positions "arm 1" and "arm 2"

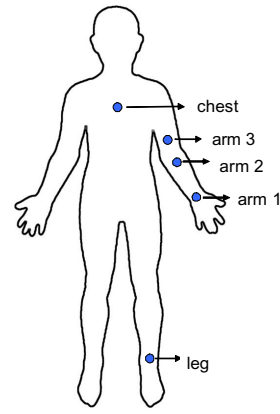

(b) measurement positions on the human body

Fig. 2. Attaching the BCC channel characterization system to a test person.

input impedance of the measurement system has to be chosen large enough in order to guarantee that this high-pass effect stops at a reasonably low-frequency.

The measurement system connected to a test person is shown in Fig. 2(a). Here the couplers consist of two parallel copper electrodes, which are isolated from each other and from the skin. The volume between the two electrodes is filled with PCB material and PVC foam.

A large number of measurements were performed in a conference room on the High Tech Campus in Eindhoven. The room is $2.70 \times 4.30 \mathrm{~m}^{2}$, has two glass walls and does not contain electronic equipment. All results presented here are for the same subject: female, height $=1.54 \mathrm{~m}$. The BCC channel was assessed for different coupling positions on the body. For five of these positions, as depicted in Fig. 2(b), results are presented in this paper. Three positions on the right arm are used ("arm 1", "arm 2" and "arm 3"), one on the chest ("chest") and one on the back of the right leg ("leg"). For the measurements the couplers were placed on the skin, however, there was no direct (galvanic) contact between the electrodes and the skin, since the electrodes were covered with isolation tape. Moreover, we considered cases where the TX and RX couplers had the same dimensions. For every experiment 255 equally spaced frequencies were tested. Each frequency was measured 255 times per experiment.

Calibration and verification tests were performed in a shielded (EMC) room. These showed that the measurements in the conference room were not influenced by interference.

\section{Propagation Loss Characterization Results}

Here we first study the propagation loss in static situations, i.e. the subject is standing on a fixed position. The influence of the coupler location on the average channel attenuation is shown for the frequency range of $100 \mathrm{kHz}$ to $60 \mathrm{MHz}$ in Fig. 3. The results are depicted for couplers of size $4 \times 4 \mathrm{~cm}^{2}$, where the electrodes are separated $1 \mathrm{~cm}$ apart. The legend of Fig. 3 reports the TX position followed by the RX location.

As expected, the propagation loss increases with increased distance between the TX and RX on the body. For propagation on the arm the loss varies between 60 and $45 \mathrm{~dB}$ from 


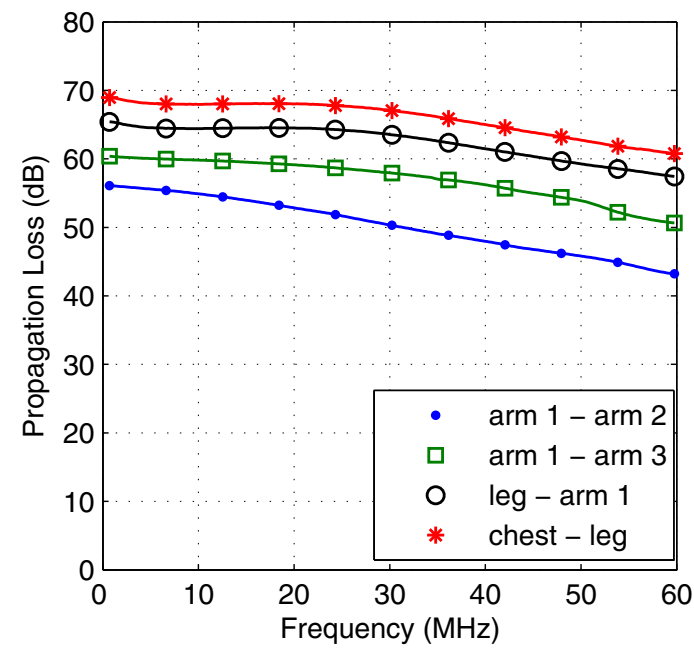

Fig. 3. Influence of transceiver positions on the propagation loss as function of frequency, for $4 \times 4 \mathrm{~cm}^{2}$ electrodes and $1 \mathrm{~cm}$ electrode separation.

the lower to higher frequencies. For the chest-leg and legarm channel an additional 10 to $15 \mathrm{~dB}$ loss occurs, but the frequency behavior is similar. The latter results show that even for large distances on the body and for communications between the front and back of the body a reasonable signal attenuation is achieved, where the latter aspect is generally challenging in RF-based WBANs. The maximum signal attenuation was typically found to be below $80 \mathrm{~dB}$. The variation of the propagation loss over frequency is likely explained by additional propagation "through the air" for the higher frequencies, yielding lower losses. This confirms previous findings reported in literature.

Moreover, the results show that the frequency dispersion of the BCC channel is very limited. Consequently, it is not very beneficial to apply multi-carrier techniques such as orthogonal frequency division multiplexing (OFDM). Also the signal detection can be relatively simple, since the low dispersion will allow for a very simple equalizer, even for large signal bandwidths of several tens of $\mathrm{MHz}$.

The reproducibility of the measurement results was confirmed by repeating several measurements with a different subject (male, height $=1.97 \mathrm{~m}$ ), on a different location, and on the same location on a different day. These results showed a typical deviation in loss of less than $5 \mathrm{~dB}$. This is illustrated in Fig. 4, which shows the results for the leg - arm 1 channel with $4 \times 4 \mathrm{~cm}^{2}$ electrodes and $1 \mathrm{~cm}$ electrode separation for the two subjects and for measurements on different days. It can be concluded from the figure that the variations in measured propagation loss are small.

Another important parameter for BCC system design is the node form factor. Typically the size of the coupler will largely govern the size of the BCC node. This size is determined by the size of the electrodes and the separation between the electrode pair forming a coupler. The influence of different electrode sizes on propagation loss is studied in Fig. 5, for the arm 1 - arm 2 channel. The distance between the couplers is kept constant to $11 \mathrm{~cm}$, independent of the the coupler size.

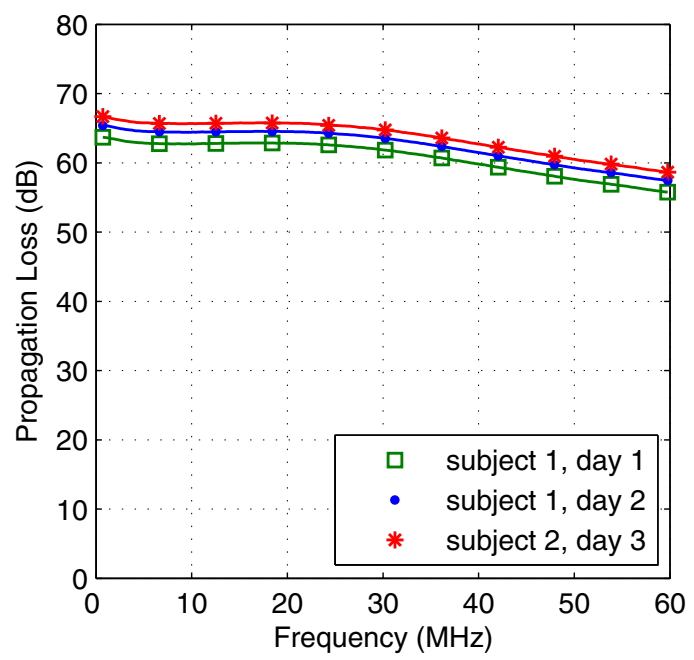

Fig. 4. Propagation loss as function of frequency for two different subjects and on different days, for $4 \times 4 \mathrm{~cm}^{2}$ electrodes, $1 \mathrm{~cm}$ electrode separation and the leg - arm 1 channel.

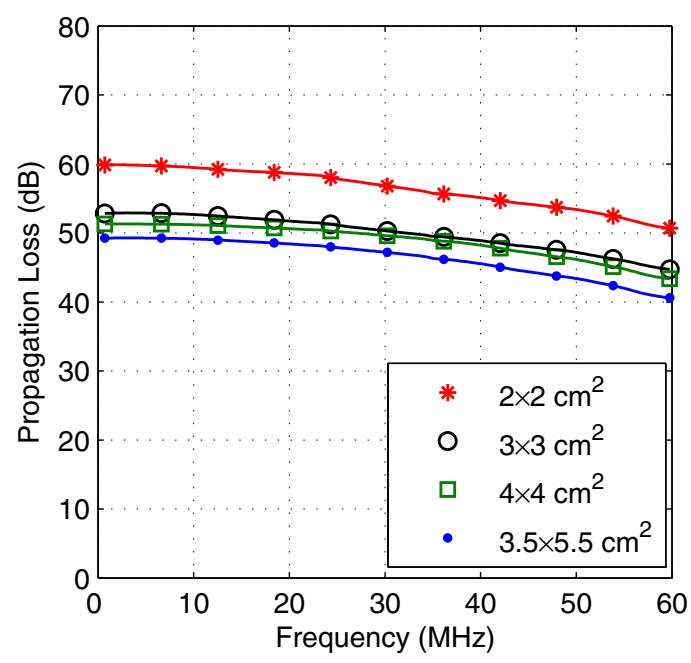

Fig. 5. Influence of electrode size on the propagation loss as function of frequency, for $1 \mathrm{~cm}$ electrode separation and the arm 1 - arm 2 channel.

We can conclude from Fig. 5 that an increased coupler size results in lower propagation loss for the whole frequency range. For the other node locations, similar results were observed. Although the $3.5 \times 5.5 \mathrm{~cm}^{2}$ electrodes show superior performance, the size might be prohibitive for WBAN implementation. Consequently the $3 \times 3 \mathrm{~cm}^{2}$ electrode size forms a nice compromise between size and resulting propagation loss.

Figure 6 illustrates the influence of electrode separation for $4 \times 4 \mathrm{~cm}^{2}$ couplers on the arm channel. It shows that overall higher separation does yield a lower propagation loss, however, not over the whole frequency range. These results might be impacted by the difference in the construction of the couplers, which are filled with PCB material and PVC foam. For small separations relatively more PCB material was present. Similar results were found for the other body locations. Overall the $2 \mathrm{~cm}$ separation showed the best performance, i.e. the lowest average propagation loss. Since too high separation between 


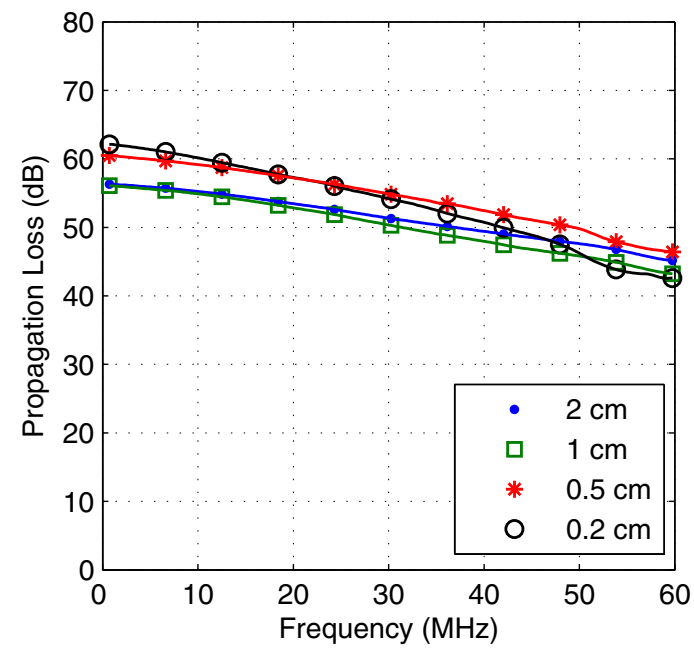

Fig. 6. Influence of electrode separation on the propagation loss as function of frequency, for $4 \times 4 \mathrm{~cm}^{2}$ electrodes and the arm 1 - arm 2 channel.

the electrodes is impracticable for WBAN nodes due to size constraints, an electrode separation of $1 \mathrm{~cm}$ is considered a suitable trade-off between size and signal attenuation. It is noted that in a practical BCC node implementation the space between the electrodes would be used to place the node electronics (for sensing/actuation and communications) and that the ground plane of the PCB could serve as one of the electrodes.

One of the advantages of $\mathrm{BCC}$ is that the communication range is limited to the body close vicinity. This is in contrast to RF systems, that generally radiate away from the body and hence provide communication coverage on and off the body. Some of the results of an assessment of this property of BCC are presented in Fig. 8. It depicts the propagation loss for the measurement setup shown in Fig. 7, where two couplers are separated $11 \mathrm{~cm}$ apart on a cardboard box. The arm of the subject was placed at different distances parallel to the couplers. The bottom and top curves in Fig. 8 represent the cases of the arm touching both couplers and no arm present,

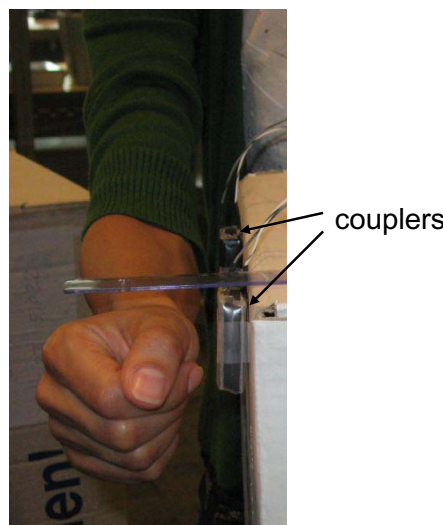

front view

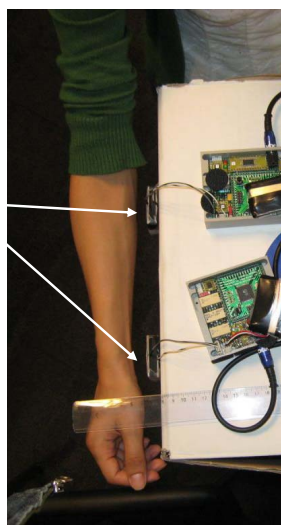

top view
Fig. 7. Measurement setup to test the influence of body separation on propagation loss. The couplers are placed $11 \mathrm{~cm}$ apart from each other and the distance of the arm to the couplers is varied.

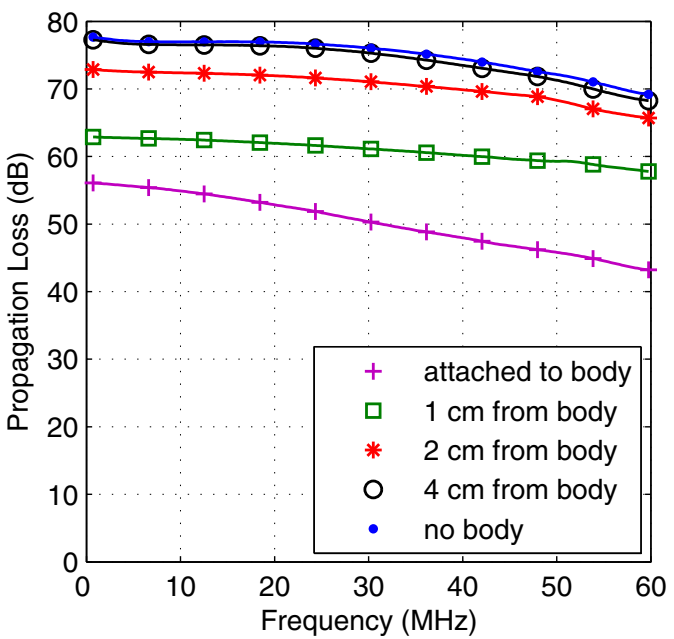

Fig. 8. Influence of body-coupler separation on the propagation loss as function of frequency, for $4 \times 4 \mathrm{~cm}^{2}$ electrode size, $1 \mathrm{~cm}$ electrode separation and the arm 1 - arm 2 channel.

respectively. There is a $25 \mathrm{~dB}$ loss difference between the two cases. When the arm is placed 1 and $2 \mathrm{~cm}$ from the couplers, an increase in loss of $7-14 \mathrm{~dB}$ and $14-22 \mathrm{~dB}$, respectively, is observed compared to the touching case. When the arm is $4 \mathrm{~cm}$ separated from the couplers, the attenuation is about equal to the case without an arm placed near to the couplers. The remaining transfer for these cases is likely mainly due to direct coupling between the TX and RX couplers caused by the relatively small distance between them.

When we compare these results with those in Fig. 3, we can clearly conclude that propagation loss off the body is orders of magnitude higher than that on the body. Hence, the communication range is indeed limited to the very close proximity of the body. This is in contrast to results found for RF-based WBAN where the communication range is typically governed by free space propagation. Consequently the communications range of these RF systems can be several meters from the body, which will likely result in interference between different WBANs.

\section{INFLUENCE OF BODY MOVEMENT}

The influence of body movement was studied to understand the variation in signal attenuation that occurs when a person is moving. To this end, measurements were performed for the subject sitting on a chair, standing, walking through the room, and standing while moving the right arm up and down, respectively. All results in this section are presented for the $4 \times 4 \mathrm{~cm}^{2}$ electrode size, $1 \mathrm{~cm}$ electrode separation and the arm 1 - arm 2 channel. For this case, both dynamic situations yielded a similar mean attenuation as the static standing scenario (see Fig. 3). For the sitting scenario, however, 10-12 dB additional channel attenuation was observed compared to the standing case. This is likely explained due to coupling interaction with the chair.

Figure 9 depicts the experimentally determined cumulative distribution function (ECDF) of the deviation from the median 


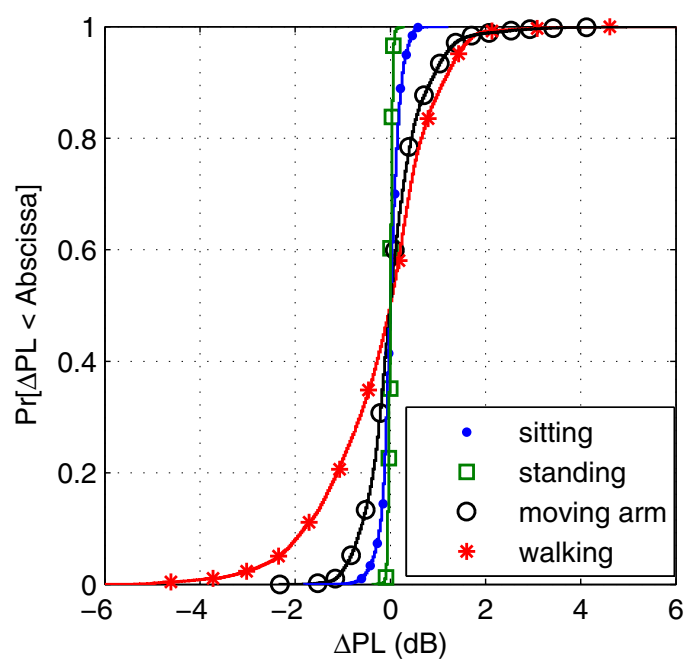

Fig. 9. ECDF of the deviation in propagation loss (in $\mathrm{dB}$ ) due to body movement, for $4 \times 4 \mathrm{~cm}^{2}$ electrode size, $1 \mathrm{~cm}$ electrode separation for the arm 1 - arm 2 channel.

of the propagation loss ( $\Delta \mathrm{PL})$ per frequency (in $\mathrm{dB}$ ), aggregated over the whole frequency range $100 \mathrm{kHz}-60 \mathrm{MHz}$.

For sitting and standing the standard deviation of the measured attenuation are very small, i.e. below $0.2 \mathrm{~dB}$. For the moving arm and walking scenario it is $0.6 \mathrm{~dB}$ and $1.14 \mathrm{~dB}$, respectively. For other channels and coupler configurations similar results were obtained. The maximum observed standard deviation was around $2.5 \mathrm{~dB}$ and occurred for the walking scenario for the largest coupler size, i.e. $3.5 \times 5.5 \mathrm{~cm}^{2}$. This can likely be explained by the fact that this coupler moved more during body movement, yielding variation in body coupling. Another possible explanation might be the capacitive coupling of the electrodes to other parts of the body while moving the arm, resulting in decreased loss for certain periods of the movement.

From the body movement results, we conclude that the signal level variability during body movements is very limited for BCC. This is in contrast to what was found for RFbased WBAN solutions, since for these systems the body shadowing does create large channel variability due to body movements, i.e. in the range of 30 to $40 \mathrm{~dB}$ [4], [5]. This poses a serious challenge for RF transceivers design, but not for $\mathrm{BCC}$ receivers.

To better understand the nature of motion-caused signal amplitude fading and to be able to simulate its impact on system performance, its distribution was investigated. The fit of different distributions (log-normal, Nakagami and Rice), commonly applied for modelling of fading in wireless channels, to the received signal amplitude over median received signal amplitude results were considered. First the data were split in groups covering $5 \mathrm{MHz}$ of bandwidth, since for larger bandwidths no coherence was observed. Then the least squares fits of the distributions were derived to find the best fit parameters to the experimental data. Figure 10 depicts the CDF fits for the moving arm and walking scenario results of Fig. 9 on a logarithmic scale $\left(20 \log _{10}(\cdot)\right.$ of the results) for

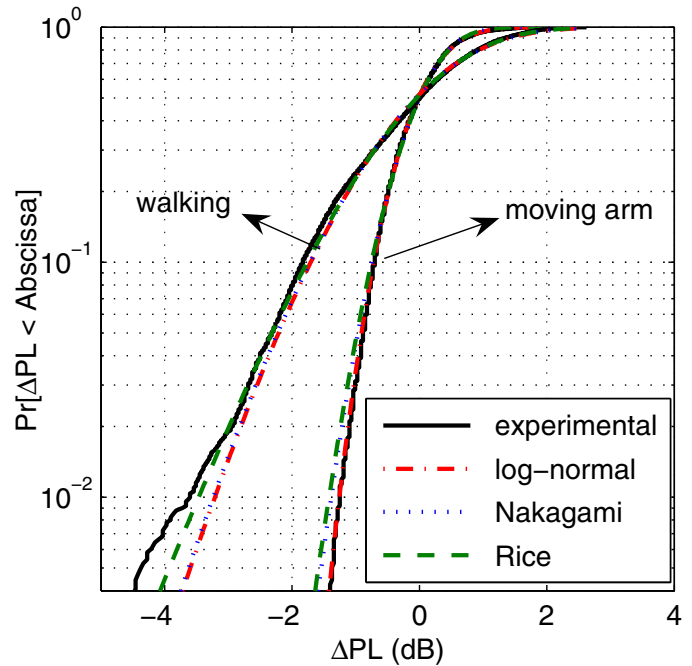

Fig. 10. ECDF of the deviation in propagation loss (in $\mathrm{dB}$ ) due to body movement together with the CDFs of the best fit theoretical distributions.

the subband $40-45 \mathrm{MHz}$.

From Fig. 10 we can observe that the log-normal distribution has the best tail-fit for the moving arm scenario, while for the walking case the Rice distribution seems to achieve a slightly better fit. Overall, for most coupler configurations and coupler positions, the center of the distribution, from 0.1 to 0.9 , is well modelled by all three distributions. The tails are of more interest, however, since they will determine error rate performance. Therefore, we concentrated on the tail fit. For this criterium it was found that the log-normal distribution showed the best overall performance.

The log-normal parameters for the amplitude fading, as presented in Fig. 10, are $\mu=0.94$ and $\sigma=0.06$ for walking, and $\mu=-0.55$ and $\sigma=0.11$ for the moving arm scenario. Averaged over all $5 \mathrm{MHz}$ subbands, the parameters are $\bar{\mu}$ equals 0.67 and -1.1 and $\bar{\sigma}$ equals 0.09 and 0.24 for the walking and moving arm scenarios, respectively.

\section{INTERFERENCE CHARACTERIZATION}

From early BCC experiments we observed that the considered frequency band is very susceptible to all kinds of electromagnetic interference. Hence, it was considered important to obtain a better understanding of the interference influence, which will enable more effective system design. To this end, interference characterization measurements were performed with a battery-fed setup, based on a portable spectrum analyzer with a probe with the same input impedance as the system presented in Section II. The setup is shown in Fig. 11(a). All presented measurements were performed with the $4 \times 4 \mathrm{~cm}^{2}$ coupler, $1 \mathrm{~cm}$ electrode separation on position "arm 1". The spectrum analyzer measurement bandwidth was $30 \mathrm{kHz}$ per measurement point. Figure 11(b) shows the characterization results for different measurement locations within a lab room.

The bottom curve depicts the result when the probe was not connected to the spectrum analyzer, showing the lowest possible level that can be measured. At low frequencies, this is mainly limited by the phase noise behavior of the spectrum 


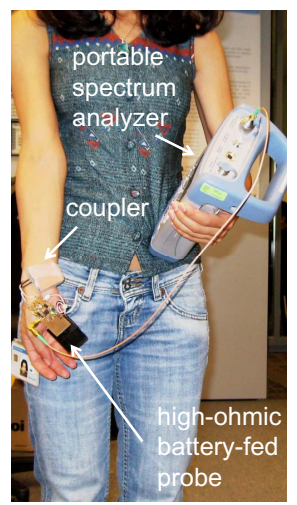

(a) interference measurement setup at "arm 1"

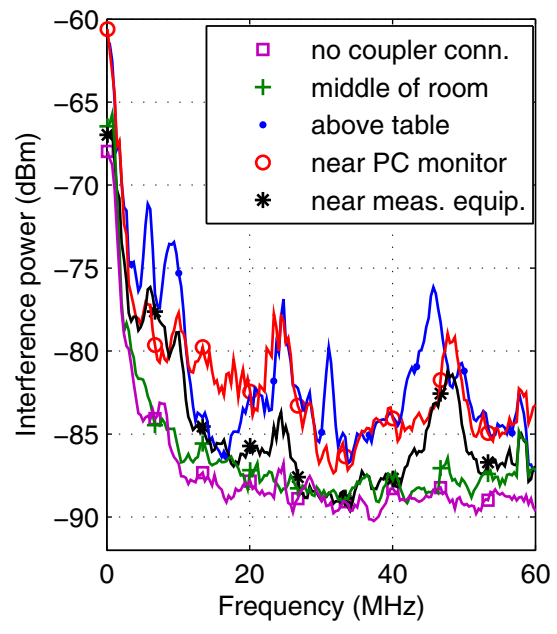

(b) measured interference powers for different measurement locations
Fig. 11. Interference characterization setup and measurement results.

analyzer. It is observed that when the subject is located in the middle of a room, the interference is almost equal to the baseline. When the subject, however, is near a monitor, other measurement equipment or places the arm above a table with metal base, the interference is severely increased. This can be explained by coupling of these devices to the body and the coupler. The table acts as "antenna" for sources of interference and in turn couples this to the electrodes/body. It is concluded from Fig. 11(b) and further measurements, that the level of interference can be considerable. Moreover, the spectral characteristics of the interference are very location dependent. For different subjects standing at the same location, the measured interference was very similar.

Due to the varying spectral properties of the interference, it would be beneficial to apply some adaptivity in the BCC data modulation scheme, especially when a wideband system would be applied. Then the system can choose the transmission mode which is at that instant least impacted by interference. This can be achieved by the use of e.g. different subbands, modulation codes or frequency hopping. If only low data rates are required, narrowband approaches might be preferable to minimize the interference impact.

\section{CONCLUSIONS}

This paper presented experimental results for the characterization of the BCC channel. A dedicated measurement system was developed, which is isolated from earth ground to simulate the WBAN scenario. The results show that channel dispersion is low and that for almost all node locations the propagation loss is well below $80 \mathrm{~dB}$. Moreover, the communications range of $\mathrm{BCC}$ is shown to be limited to the close vicinity of a human body, allowing the whole frequency band to be reused by all WBANs. Body movements were shown to result in only small variations in channel attenuation, i.e. the maximum observed standard deviation was $2.5 \mathrm{~dB}$. The received signal magnitude fading due to motion was shown to be well modelled by the log-normal distribution.
The propagation loss values found in this work are similar to those observed for RF-based WBANs in literature, see e.g. [5]. However, due to the much lower carrier frequency, the lower channel dispersion and the much lower fading due to body movement, BCC can result in a much simpler and more robust communications solution with a lower power consumption than traditional RF-based WBANs. Hence, this leads us to the conclusion that BCC is a promising basis for future WBANs.

From a study on the optimal form factor of the coupler, it was concluded that increasing the separation of the parallel electrodes and increasing the coupler size decreases the propagation loss. Moreover, it was concluded that $3 \times 3 \mathrm{~cm}^{2}$ electrodes with $1 \mathrm{~cm}$ separation achieve a nice tradeoff between propagation loss and size of the BCC node. Finally, interference results showed that capacitive coupling is susceptible to interference from other electronic equipment, the characteristics of which largely depend on the location.

Although the current results allow us to drawn qualitative conclusions about the capacitive BCC channel, this work (and previous literature) is not exhaustive, as statistically relevant populations are not yet achieved. More tests are hence required to draw solid quantitative conclusions. Such further refined understanding will allow one to come to an efficient BCC system design.

\section{ACKNOWLEDGEMENTS}

The authors acknowledge the useful discussions with their Philips Research colleagues Alberto Fazzi, Steven Corroy, Karin Klabunde and Heribert Baldus.

\section{REFERENCES}

[1] T. Falck, H. Baldus, J. Espina, and K. Klabunde, "Plug 'n Play simplicity for wireless medical body sensors," MONET, vol. 12, no. 2-3, 2007.

[2] T.G. Zimmerman, "Personal Area Networks (PAN): Near-field intrabody communication," M.S. thesis, MIT Media Laboratory, 1995.

[3] N. Matsushita, S. Tajima, Y. Ayatsuka, and J. Rekimoto, "Wearable key: Device for personalizing nearby environment," in Proc. Int. Wearable Comput. Symp., Oct. 2000, pp. 119-126.

[4] D. Neirynck, C. Williams, A. Nix, and M. Beach, "Wideband channel characterisation for body and personal area networks," in Proc. BSN Workshop, 2004, pp. 41-43.

[5] Y.I. Nechayev, P.S. Hall, C.C. Constantinou, Y. Hao, A. Alomainy, R. Dubrovka, and C.G. Parini, "On-body path gain variations with changing body posture and antenna position," in Proc. IEEE AP-S International Symposium, 2005, vol. 1B, pp. 731-734.

[6] T.-Y. Lin and Y.-C. Tseng, "Collision analysis for a multi-Bluetooth picocells environment," IEEE Commun. Lett., vol. 7, no. 10, Oct. 2003.

[7] P. Popovski, H. Yomo, and R. Prasad, "Dynamic adaptive frequency hopping for mutually interfering wireless personal area networks," IEEE Trans. on Mobile Computing, vol. 5, no. 8, Aug. 2006.

[8] S.-J. Song, N. Cho, S. Kim, J. Yoo, and H.-J. Yoo, "A 2 Mb/s wideband pulse transceiver with direct-coupled interface for human body communications," in Proc. IEEE ISSCC, Feb. 2006, pp. 558-559.

[9] M.S. Wegmueller, M.O. Norbert Felber, N. Kuster, and W. Fichtner, "Galvanical coupling for data transmission through the human body," in Proc. Instrum. Meas. Technol. Conf., Apr. 2006, pp. 1686-1689.

[10] N. Cho, J. Yoo, S.-J. Song, J. Lee, S. Jeon, and H.-J.Yoo, "The human body characteristics as a signal transmission medium for intrabody communication," IEEE Trans. on MTT, vol. 55, no. 5, May 2007.

[11] J.A. Ruiz, Jiang Xu, and S. Shimamoto, "Propagation characteristics of intra-body communications for body area networks," in Proc. 3rd IEEE CCNC conference, Jan. 2006, vol. 1, pp. 509-513.

[12] J.A. Ruiz and S. Shimamoto, "Statistical modeling of intra-body propagation channel," in Proc. IEEE Wireless Communications and Networking Conference, Mar. 2007, pp. 2063-2068. 